\title{
Quantum-Fluctuation-Driven Crossover from a Dilute Bose-Einstein Condensate to a Macrodroplet in a Dipolar Quantum Fluid
}

\author{
L. Chomaz, ${ }^{1}$ S. Baier, ${ }^{1}$ D. Petter, ${ }^{1}$ M. J. Mark, ${ }^{1,2}$ F. Wächtler, ${ }^{3}$ L. Santos, ${ }^{3}$ and F. Ferlaino ${ }^{1,2, *}$ \\ ${ }^{1}$ Institut für Experimentalphysik, Universität Innsbruck, Technikerstraße 25, 6020 Innsbruck, Austria \\ ${ }^{2}$ Institut für Quantenoptik und Quanteninformation, Österreichische Akademie der Wissenschaften, \\ 6020 Innsbruck, Austria \\ ${ }^{3}$ Institut für Theoretische Physik, Leibniz Universität Hannover, \\ Appelstrasse 2, 30167 Hannover, Germany \\ (Received 22 July 2016; revised manuscript received 15 September 2016; published 22 November 2016)
}

In a joint experimental and theoretical effort, we report on the formation of a macrodroplet state in an ultracold bosonic gas of erbium atoms with strong dipolar interactions. By precise tuning of the $s$-wave scattering length below the so-called dipolar length, we observe a smooth crossover of the ground state from a dilute Bose-Einstein condensate to a dense macrodroplet state of more than $2 \times 10^{4}$ atoms. Based on the study of collective excitations and loss features, we prove that quantum fluctuations stabilize the ultracold gas far beyond the instability threshold imposed by mean-field interactions. Finally, we perform expansion measurements, showing that although self-bound solutions are prevented by losses, the interplay between quantum stabilization and losses results in a minimal time-of-flight expansion velocity at a finite scattering length.

DOI: 10.1103/PhysRevX.6.041039

\section{INTRODUCTION}

The extraordinary success of ultracold quantum gases largely stems from the simplicity with which the physics at the many-body level can be controlled and described, allowing access to a wide range of theoretical models of general interest [1]. Notably, the actual many-body interactions are often very well captured via simple mean-field (MF) potentials, proportional to the local particle density $n$ and accounting for the average mutual effect of all neighboring particles [1]. Moreover, short-ranged interactions, even if complex or unknown, can be simply accounted for via a contact potential and parametrized by the sole $s$-wave scattering length $a_{s}$, which in turn can be widely tuned by means of Feshbach resonances (FRs) [2]. The MF treatment of a Bose gas leads to the celebrated Gross-Pitaevskii equation (GPE) and Bogoliubov-de Gennes (BdG) spectrum of collective modes, which are very powerful in describing the physics of an ultracold bosonic gas: its ground-state properties as a Bose-Einstein condensate (BEC), as well as its dynamics [1].

Beyond the great achievements of dilute gases as a test bed for MF theories, the quest for beyond-MF effects has triggered great interest in the ultracold community. The

*Francesca.Ferlaino@uibk.ac.at

Published by the American Physical Society under the terms of the Creative Commons Attribution 3.0 License. Further distribution of this work must maintain attribution to the author(s) and the published article's title, journal citation, and DOI.
Subject Areas: Atomic and Molecular Physics, Quantum Physics general question of how the many-body ground state of bosons is modified by quantum fluctuations (QFs) of elementary excitations was first addressed by Lee, Huang, and Yang (LHY) in the 1950s [3]. The so-called LHY term, which accounts for the first-order correction to the condensate energy, scales for a contact-interacting gas as $a_{s} n \sqrt{n a_{s}^{3}}$. While in the weakly interacting regime the effect of QFs is negligible and difficult to isolate from MF contributions, it can be sufficiently amplified by increasing $a_{s}$ via a FR. Based on this concept, recent experiments with alkali have observed clear shifts of the BdG spectrum and equation of state caused by the LHY term in strongly interacting Fermi [4-6] and Bose gases [7,8].

While in these measurements the LHY correction does not modify the qualitative behavior of the gas, it has been recently pointed out [9] that, in systems with competing interactions of different origin, the MF interaction can be made small and the LHY term dominant, so that the latter dictates the physics of the system, even in weakly interacting gases. In this regime, a novel phase of matter is expected to appear, namely, a liquidlike droplet state. For purely contactinteracting gases, this situation is hard to realize since it would require, for instance, Bose-Bose mixtures with coincidental overlapping FRs [9]. In contrast, dipole-dipole interaction (DDI) genuinely offers this possibility in a single-component atomic gas by competing with the isotropic MF contact interaction $[10,11]$. In the pure MF picture, a paradigm of the competition between DDI and contact interaction is embodied by the ability of quenching a dipolar BEC to collapse by varying $\varepsilon_{\mathrm{dd}}=a_{\mathrm{dd}} / a_{s}$, where 
$a_{\mathrm{dd}}=\mu_{0} \mu^{2} m / 12 \pi \hbar^{2}$ is a characteristic length set by the DDI, with $m$ the mass and $\mu$ the magnetic moment of the atoms $[12,13]$. Here, $\hbar$ stands for the reduced Planck constant and $\mu_{0}$ for the vacuum permeability. In general, because of the special geometrical tunability of DDI with the external trapping potential and dipole orientation, the stability and phase diagram remarkably depend on $\lambda=\nu_{\|} / \nu_{\perp}$, where $\nu_{\|}\left(\nu_{\perp}\right)$ is the trapping frequency along (perpendicular to) the dipole orientation $[11,12,14]$.

In parallel, recent breakthrough experiments with an oblate dysprosium (Dy) dipolar BEC $(\lambda>1)$ have shown that when quenching up $\varepsilon_{\mathrm{dd}}$, the system, instead of collapsing, forms a metastable state of several small droplets $[15,16]$. This observation has triggered an intense debate on the nature of such a state and its underlying stabilization mechanism [17-23]. Eventually, Dy experiments indicated QFs as the origin of the stabilization [16], which were quickly confirmed by theoretical works [20-22]. Furthermore, these theoretical studies highlight the richness of the dipolar-gas phase diagram, in which a dilute-BEC, a multidroplet, and a single-droplet phase are found for distinct $a_{s}, a_{\mathrm{dd}}$, atom number $N$, and $\lambda$. Up to now, droplet physics has only been investigated in a single setup, using Dy BEC and exploring a specific region of the phase diagram: In the considered pancake geometry $(\lambda>1)$, multiple stable solutions - single droplet or multidropletcoexist, resulting in the formation of variable mesoscopic assemblies of a small droplet in the experiments.

In the present work, we (i) demonstrate the generality of droplet physics, by using a dipolar BEC of erbium (Er) atoms [24], (ii) quantitatively investigate the specific role played by QFs in dipolar systems, and (iii) explore a pristine region of the phase diagram, studying a cigar-shaped geometry $(\lambda \ll 1)$, and observe the crossover from a dilute BEC to a single macrodroplet state when increasing $\varepsilon_{\mathrm{dd}}$, as predicted in Refs. [21,22]. Given the complexity of the physics at play, we combine distinct measurements, based on the observations of the density distributions, collectives excitations, expansion dynamics, and lifetime of the dipolar quantum gas, which together offer a comprehensive picture of droplet physics. The exquisite control of the scattering length gained in our experiment, together with a direct comparison to parameterfree simulations including QF effects, ultimately enable us to depict in which way QFs dictate the physics at play, beyond proving their crucial stabilizing role.

\section{EXPERIMENTAL PROCEDURES}

The atomic properties of Er offer a privileged platform to explore a variety of interaction scenarios. Besides its strongly magnetic character and its many FRs [25], Er has several stable isotopes. This feature adds an important flexibility in terms of the choice of the background $a_{s}$ [26]. In our early work on Er BECs, we employed the ${ }^{168} \mathrm{Er}$ isotope, which has a background $a_{s}$ about twice as large as the dipolar length, $a_{\mathrm{dd}}=65 a_{0}[27,28]$.

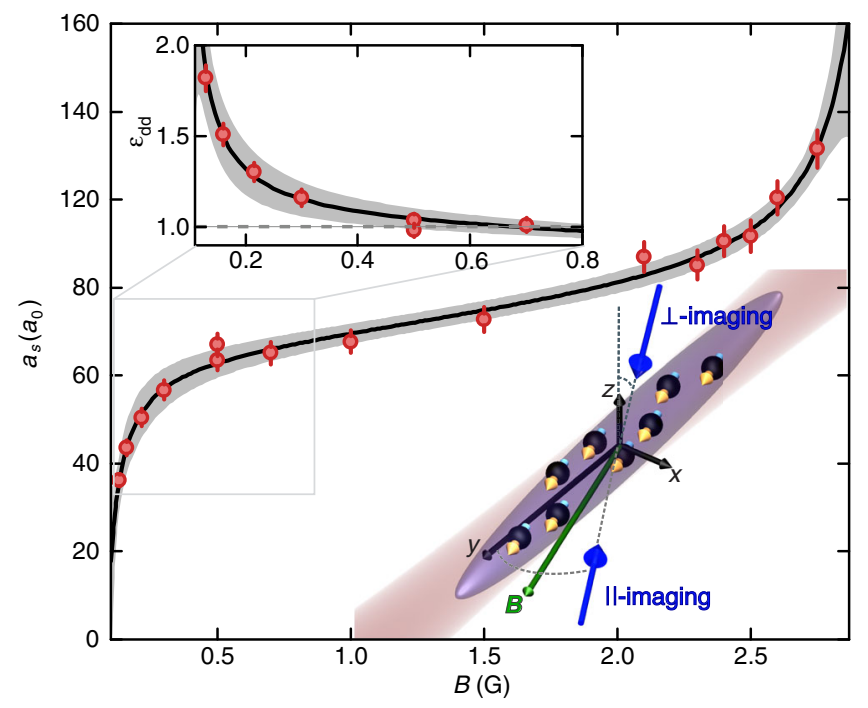

FIG. 1. Scattering length in ${ }^{166}$ Er. $a_{s}$ as a function of $B$. The data points (circles) are extracted from spectroscopic measurements in a lattice-confined gas and the solid line is a fit to the data with its statistical uncertainty (gray shaded region [30]). Upper inset: Zoom-in of $\varepsilon_{\mathrm{dd}}$ as a function of $B$. The gray dashed line marks $\varepsilon_{\mathrm{dd}}=1$; see also the other figures. The lower inset illustrates the geometry of our experimental setup, the relevant axes $(x, y, z)$, the optical-dipole-trap beam (shaded region), the magnetic field orientation (green arrow) along which the dipoles are aligned, and the $\|$ - and $\perp$-imaging view axes (blue arrows). The dashed lines picture the small angles of these axes to $y$ and $z$ [30].

In the work reported here, we produce and use a BEC of ${ }^{166} \mathrm{Er}$ in the lowest internal state. This isotope provides us with two major advantages. First, its background $a_{s}$ is comparable to its dipolar length, $a_{\mathrm{dd}}=65.5 a_{0}$, realizing $\varepsilon_{\mathrm{dd}}=a_{\mathrm{dd}} / a_{s} \approx 1$ without the need of Feshbach tuning. Second, ${ }^{166}$ Er features a very convenient FR at ultralow magnetic-field values $B$. To precisely map $a_{s}$ as a function of $B$, we use a spectroscopic technique based on the measurement of the energy gap of the Mott insulator state in a deep three-dimensional optical lattice [28,29]. A detailed description is given in the Supplemental Material [30]. Between 0 and $3 \mathrm{G}$, we observe a smooth variation of $a_{s}$, which results from two low-lying FRs whose centers are fitted to 0.05(5) and 3.0(1) G, respectively; see Fig. 1. This feature gives easy access into the $\varepsilon_{\mathrm{dd}}>1$ regime, allowing variation of $\varepsilon_{\mathrm{dd}}$ from 0.70 (2) to 1.58 (18) by changing $B$ from 2.5 to $0.15 \mathrm{G}$; see Fig. 1 upper inset. By fitting our data [2], we extract $a_{s}(B)$ valid for $B$ in the $[0.15,2.5]-G$ range, which we use throughout this paper [30].

We achieve Bose-Einstein condensation of ${ }^{166} \mathrm{Er}$ using an all-optical scheme very similar to Ref. [27] with cooling parameters optimized for ${ }^{166} \mathrm{Er}$ [30]. In short, we drive forced evaporative cooling at a magnetic field $B=1.9 \mathrm{G}$, corresponding to $a_{s}=81(2) a_{0}\left[\varepsilon_{\mathrm{dd}}=0.81(2)\right]$. In this phase, $B$ is oriented along the vertical $z$ axis. At the end of the evaporation, we obtain a BEC of $N=1.2 \times 10^{5}$ atoms with a condensed fraction above $80 \%$. 
To reach the $\lambda \ll 1$ regime, we slowly modify, in the last step of the evaporation, the confining potential to the final cigar shape, with typical frequencies $\left(\nu_{x}, \nu_{y}, \nu_{z}\right)=$ [156(1), 17.2(4), 198(2)] Hz. Simultaneously, we decrease $B$ to $0.8 \mathrm{G}\left[a_{s}=67(2) a_{0}\right]$ and then change the magneticfield orientation to the weak trapping axis $(y)$ while keeping its amplitude constant [30]. Finally, we ramp $B$ to the desired target value (and equivalently $a_{s}$ ) in $t_{r}$ [30], hold for a time $t_{h}$, and perform absorption imaging of our gas after a time-of-flight (TOF) of $t_{\text {TOF }}$. Two imaging setups are used in order to measure the density distribution integrated either along the dipoles (II imaging) or perpendicular to them ( $\perp$ imaging) [30]. Figure 1 (lower inset) illustrates the final geometry of our system with $\nu_{\|}=\nu_{y}, \nu_{\perp}=\sqrt{\left(\nu_{x}^{2}+\nu_{z}^{2}\right) / 2}$, giving $\lambda=0.097(3)$, and defines the relevant axes.

Here, we explore the properties of the system when the repulsive MF contact interaction is weakened enough to be overcome by the DDI $\left(\lambda \ll 1, \varepsilon_{\mathrm{dd}}>1\right)$, after adiabatically changing $\left(t_{r} \geq 45 \mathrm{~ms}\right)$ or quenching $\left(t_{r}=10 \mathrm{~ms}\right) a_{s}$ to its target value [30]. For $t_{r} \geq 45 \mathrm{~ms}$, the system evolves following its ground state and gives access to the slow dynamics, whereas for the $t_{r}=10 \mathrm{~ms}$ case, we can probe the fast dynamics and study the relaxation towards an equilibrium. The key question is whether QFs protect the system from collapsing. Indeed, in this regime, the MF treatment would imply that the attractive BEC becomes unstable, leading to a twofold dramatic consequence [1]. First, some modes of the BdG spectrum acquire complex frequencies. Second, in a trap, the density distribution of the cloud undergoes a marked change on short time scales $\left(\leq 1 / \nu_{\perp}\right)$, described as a "collapse", which can develop into a rapid loss of coherence [12,31], and pattern formations, such as anisotropic atom bursts ("bosenova") and special $d$-wave-type structures, as observed in rubidium [32] and dipolar gases of chromium [12,13], respectively. This fast dynamics has been proved to be well encompassed by GPE simulation $[13,14,33]$.

\section{DENSITY DISTRIBUTION}

In a first set of experiments, we study the stability of our dipolar Er BEC by probing the evolution of the TOF density distribution for different $a_{s}$. Figures 2(a)-2(c) show the absorption images acquired with $\|$ imaging [Figs. 2(a)-2(c)] and the corresponding central cuts $(x=0)$ of the 2D column density profiles [Fig. 2(d)]. In striking contrast to the MF predictions, we observe that the system remains stable for $a_{s}$ well below $a_{\mathrm{dd}}$, with a central coherent core surviving for times much longer than $1 / \nu_{\perp}$ (from several tens to hundreds of $\mathrm{ms}$ ). The density distribution does not exhibit any special patterns, which is typical of a collapsing cloud $[12,13,32]$.

For $a_{s}>a_{\mathrm{dd}}$ [Fig. 2(a)], the density distribution of the gas shows good agreement with the MF Thomas-Fermi (TF) profile on top of a broad Gaussian distribution,
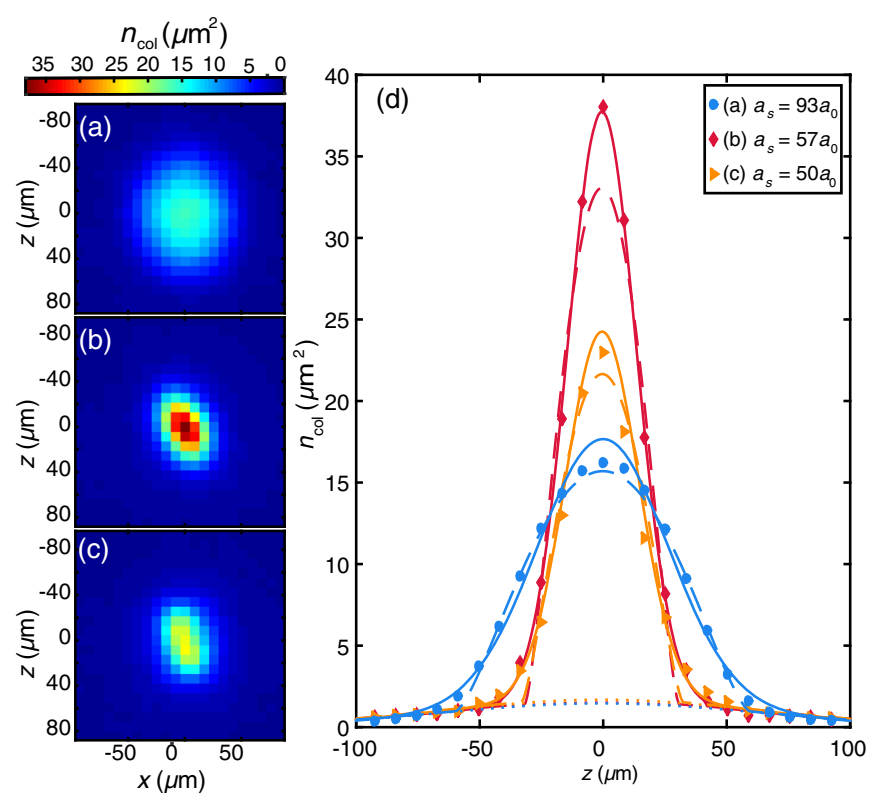

FIG. 2. Density profiles in the BEC-to-droplet crossover. (a)-(c) 2D column density distributions probed with $\|$ imaging and (d) corresponding central cuts along the $x=0$ line (dots) for $t_{r}=10 \mathrm{~ms}, t_{h}=6 \mathrm{~ms}\left(>1 / \nu_{\perp}\right)$, and different $a_{s}$ (see legend). Each distribution is obtained by averaging four absorption images taken after $t_{\mathrm{TOF}}=27 \mathrm{~ms}$. In (d), the lines show the central cuts of the $2 \mathrm{D}$ bimodal fit results, the solid (dashed) lines showing the two-Gaussian (MF-TF plus Gaussian) distributions and the dotted lines the corresponding broad thermal Gaussian part.

accounting for the thermal atoms; see Fig. 2(d), dashed lines. When lowering $a_{s}$ below $a_{\mathrm{dd}}$ [Fig. 2(b)], we observe a sharpening of the central core, whose profile starts to deviate from the MF-TF shape (see Ref. [30] for a quantitative description). When decreasing $a_{s}$ even further [Fig. 2(c)], a similar bimodal structure holds on although the dense core loses atoms. Because of the high density reached, three-body (3B) collisions regulate the lifetime of the central core; see discussion below and Ref. [23]. We note that we observe a similar qualitative behavior of the density distribution when using an adiabatic ramp of $a_{s}$. However, the importance of the central peak is reduced as, in this case, losses already set in during the ramp.

In contrast with the behavior of the central core, the distribution of the thermal atoms, encompassed by the broad Gaussian function of the bimodal fits [see Fig. 2(d), dotted lines], remains mainly unaffected by the change of $a_{s}$, highlighting an absence of significant heating and population transfer, and thus an apparent decoupling of the evolution of the coherent and thermal parts.

For further analysis, we fit the data to a bimodal distribution made of the sum of two Gaussian functions, as it offers a smaller residue than the fit to the MF-TF distribution for $a_{s} \lesssim 70 a_{0}$; see Fig. 2(d). We note that the beyond-MF effects on the density profile are expected to be more sophisticated than a Gaussian shaping. However, 
theoretical studies show good agreement between the Gaussian ansatz and the full numerical solution for our parameter range $[22,23]$.

Being a smoking gun for long-range phase coherence, the survival of a bimodal profile in the TOF distribution far beyond the MF instability threshold points to a persistent coherent behavior. This absence of a collapse advocates the outbreak of an additional stabilization mechanism, which we now further investigate by probing global properties of the gas.

\section{COLLECTIVE OSCILLATION}

In a second set of experiments, we unveil the origin of the stabilization mechanism by studying the elementary excitations of the coherent cloud. This is a very powerful probe of the fundamental properties in quantum degenerate gases [1,34]. In particular, collapse is intimately related to the softening of some collective modes at the MFinstability threshold. We focus here on the axial mode, which is the lowest-lying excitation in the system above the dipole mode. It corresponds to a collective oscillation of the condensate length along $y\left(R_{\|}\right)$with frequency $\nu_{\text {axial }}$. The axial oscillation comes along with a smaller-amplitude oscillation of the radial sizes in phase opposition; see Fig. 3(a). As a result, this mode has a mixed character between a compression and a surface mode [1]. The compression character is particularly relevant since it involves a change in the density and it is therefore sensitive to the LHY corrections [35].

We excite the axial mode either by ramping $B$ during the final preparation stage or by transiently increasing the power of the vertical optical dipole trap beam, after ramping $B$ to $B_{f}$. Here, $\nu_{\|}$is abruptly changed from $17 \mathrm{~Hz}$ to typically $21 \mathrm{~Hz}$, kept at this higher value for $8 \mathrm{~ms}$, and finally set back to $17 \mathrm{~Hz}$. Following the excitation, we let the cloud evolve for a variable $t_{h}$ and image its TOF density distribution with $\perp$ imaging. To extract $\nu_{\text {axial }}$, we probe the axial width $R_{\|}$of the central coherent component of the gas [30] with $t_{h}$ and fit it to a damped sine; see inset of Fig. 3(b).

Figure 3 shows the observed $\nu_{\text {axial }}$ normalized to the trapping frequency $\nu_{\|}$[36] as a function of $a_{s}$ for adiabatic [Fig. 3(b)] and nonadiabatic [Fig. 3(c)] ramps. Both cases exhibit a similar qualitative behavior. For $a_{s}>a_{\mathrm{dd}}$, the oscillations show a smooth dependence on $\varepsilon_{\mathrm{dd}}$, with $\nu_{\text {axial }}$ increasing by about $5 \%$ with an average value of $1.70 \nu_{\|}$ [37]. When lowering $a_{s}$, the oscillation of the coherent part remains visible well below the $\varepsilon_{\mathrm{dd}}=1$ threshold and $\nu_{\text {axial }}$ exhibits a marked increase. $\nu_{\text {axial }} / \nu_{\|}$grows up to 2.6(1) at $a_{s}=54 a_{0}$ for $t_{r}=100 \mathrm{~ms}$ [Fig. 3(b)]. For $t_{r}=10 \mathrm{~ms}$ [Fig. 3(c)], $\nu_{\text {axial }} / \nu_{\|}$first increases similarly to the adiabatic case [Fig. 3(b)], reaches a maximum of $\sim 2.13(7)$ at $57 a_{0}$ $\left(\varepsilon_{\mathrm{dd}}=1.15\right)$, and finally decreases for even smaller $a_{s}$ (open squares). The latter behavior can be explained by the fact that the larger quenches in the interaction excites

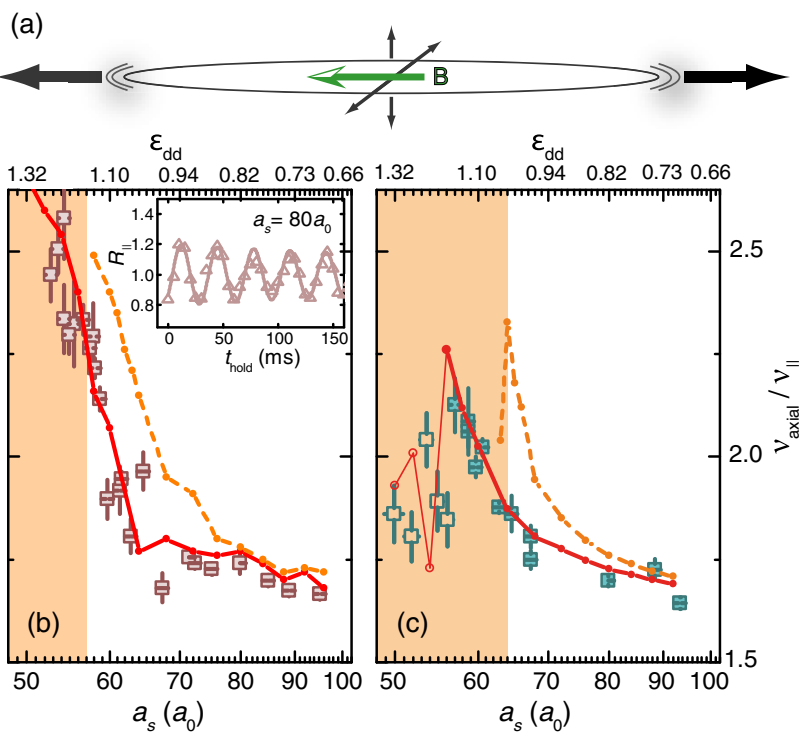

FIG. 3. Axial mode. (a) Illustration of the axial mode in our experimental setup. The black arrows sketch the oscillations of the widths of the coherent gas along the characteristic axes of the trap, with weights indicating their relative amplitudes. (b), (c) Measured $\nu_{\text {axial }} / \nu_{\|}$(squares) as a function of $a_{s}$ together with the theoretical predictions, including (solid line) or not (dashed lines) the LHY term for $t_{r}=100 \mathrm{~ms}$ (b) and $t_{r}=10 \mathrm{~ms}$ (c). Theoretical predictions are obtained from RTE (see text) for $a_{s}$ varied from $50 a_{0}$ to $95 a_{0}$. In the MF case, predictions fail for $a_{s} \leq a_{c}$ (orange area) due to the occurrence of the collapsing dynamics which rules out the collective excitation picture. $a_{c}=57 a_{0}\left[a_{c}=64 a_{0}\right]$ in (b) [(c)]. In (c), $\nu_{\text {axial }}$ cannot be reliably extracted for quenches to $a_{s} \leq 56 a_{0}$, nor from the experiment (open squares) or from the LHY theory (open circles, thin line). The inset in (b) exemplifies a measurement of $R_{\|}$(triangles) and its fit to a damped sine (solid line) for $a_{s}=80 a_{0}$. We typically fit 4-5 oscillations for all our $a_{s}$.

additional high-energy modes while it drives the system away from the linear response regime [38]. A similar behavior is found from our theory predictions including the LHY term (see below), thus highlighting a qualitative agreement even in this small- $a_{s}$ range.

\section{THEORY}

To account for our observation and discern between the MF instability picture and QF mechanisms, we develop a beyond-MF treatment of our system at $T=0$. The coherent gas is described here by means of the generalized nonlocal nonlinear-Schrödinger equation (gNLNLSE), which includes the first-order correction from QF effects, i.e., the LHY term, and 3B loss processes. The gNLNLSE reads as $[20,23]$

$$
i \hbar \frac{\partial \psi}{\partial t}=\left[\hat{H}_{0}+\mu_{\mathrm{MF}}\left(n, \epsilon_{d d}\right)+\Delta \mu\left(n, \epsilon_{d d}\right)-i \hbar \frac{L_{3}}{2} n^{2}\right] \psi,
$$


where $\hat{H}_{0}=\left[\left(-\hbar^{2} \Delta\right) / 2 m\right]+V(\boldsymbol{r})$ is the noninteracting Hamiltonian and $V(\boldsymbol{r})=2 \pi^{2} m \sum_{\eta=x, y, z} \nu_{\eta}^{2} \eta^{2}$ the harmonic confinement. The MF chemical potential, $\mu_{\mathrm{MF}}\left[n(\mathbf{r}), \epsilon_{d d}\right]=$ $g n(\mathbf{r})+\int d^{3} r^{\prime} V_{\mathrm{dd}}\left(\mathbf{r}-\mathbf{r}^{\prime}\right) n\left(\mathbf{r}^{\prime}\right)$, results from the competition between short-range interactions, controlled by the coupling constant $g=4 \pi \hbar^{2} a_{s} / m$, and the DDI term with $V_{\mathrm{dd}}(\mathbf{r})=\left[\left(\mu_{0} \mu^{2}\right) / 4 \pi r^{3}\right]\left(1-3 \cos ^{2} \theta\right)$ and $\theta$ the angle sustained by $\mathbf{r}$ and the dipole moment $\mu$. Here, $n(\mathbf{r})=|\psi(\mathbf{r})|^{2}$. The beyond-MF physics is encoded in the LHY term, leading to an additional repulsive term in the chemical potential, $\Delta \mu\left(n, \epsilon_{d d}\right)=[32 /(3 \sqrt{\pi})] g n \sqrt{n a^{3}} F\left(\epsilon_{d d}\right)$. The function $F\left(\epsilon_{d d}\right)=\frac{1}{2} \int d \theta_{k} \sin \theta_{k}\left[1+\epsilon_{d d}\left(3 \cos ^{2} \theta_{k}-1\right)\right]^{5 / 2}$ is obtained from the LHY correction in homogeneous $3 \mathrm{D}$ dipolar BECs [39-41] using local-density approximation [42]. The last non-Hermitian term in Eq. (1) accounts for 3B loss processes [43]. In our calculations, we use the experimentally determined values of the $3 \mathrm{~B}$ recombination rate of the condensate $L_{3}\left(a_{s}\right)$ [30].

As discussed in Refs. [22,23], due to the repulsive LHY term, Eq. (1) sustains stable ground-state solutions for any $a_{s}$ and $\lambda$. For pancake traps $(\lambda>1)$, the solution of Eq. (1) is not unique. The phase diagram reveals three types of solutions: the one of a dilute BEC, a single droplet solution, and a third one, which separates the previous two phases, that corresponds to a metastable region of multidroplet states. The latter has been observed in Dy experiments [15]. However, the single-droplet solution appears difficult to access because of the overhead multidroplet state and the stringent 3B loss mechanisms. Remarkably, in cigar-shaped traps $(\lambda<1)$, Eq. (1) has only one possible solution. In the $\varepsilon_{\mathrm{dd}}$ parameter space, the corresponding wave function exhibits a smooth crossover from a dilute BEC to a single, high-density, macrodroplet solution for increasing $\varepsilon_{\mathrm{dd}}$. It is worth noting that the crossover physics, e.g., the formation and lifetime of the droplet state, is expected to crucially depend on the 3B collisional processes. In the following, we concentrate on the $\lambda<1$ case, which corresponds to our experimental setting.

The continuous and smooth change of the static properties of the system with increasing $\varepsilon_{\mathrm{dd}}$ is consistent with our observations on the evolution from a dilute into an high-density state; see Fig. 2.

Based on Eq. (1), we theoretically investigate the dynamics of the coherent gas. In order to compare as close as possible the theory to our experimental results, we precisely account for the experimental sequence by performing real-time evolution (RTE) starting from the ground state of Eq. (1) at $a_{s}=67 a_{0}$ with $N=1.2 \times 10^{5}$ atoms. We simulate a linear ramp in $a_{s}$ from $67 a_{0}$ to a variable final value of $a_{s}$ in $t_{r}$, followed by a compression of the axial trap from $\nu_{\|}=17.3$ to $21 \mathrm{~Hz}$ during $8 \mathrm{~ms}$. We then record the axial width from the standard deviation of $n(\boldsymbol{r})$, $\sigma_{y}=\sqrt{\left\langle y^{2}\right\rangle}$, as a function of the subsequent holding time. The evolution of $\sigma_{y}$ is well fitted by a sinusoidal function, whose frequency constitutes our theoretical prediction of $\nu_{\text {axial }}$.

In Fig. 3, we present our calculations with and without the LHY term. The MF simulations reveal a critical scattering length $a_{\mathrm{c}}<a_{\mathrm{dd}}$ below which the system collapses, thus ruling out the collective mode excitation picture for $a_{s}<a_{c}$. This is in qualitative disagreement with the experimental observations. Moreover, for decreasing $a_{s} \geq a_{c}$, the MF predictions of $\nu_{\text {axial }}$ are sizably shifted compared to our measurements. In contrast, the experiment shows an excellent match with the theory including the LHY term, thus ruling out the MF scenario and demonstrating the crucial role played by QFs in stabilizing the system. Then, QFs qualitative modify the phase diagram and drive the formation of a special coherent state, namely, a single macrodroplet [20-23]. The lowering of $a_{c}=57 a_{0}$ found in Fig. 3(b) compared to Fig. 3(c) $\left(a_{\mathrm{c}}=64 a_{0}\right)$ arises from the more stringent interplay between QFs and 3B losses within this longer ramp, both mechanisms being able to drive the system out of the instability region.

\section{LOSS DYNAMICS}

To further investigate the respective roles of $3 \mathrm{~B}$ losses and QFs, we study the time evolution of the atom number of both the central core $\left(N_{\text {core }}\right)$ and thermal $\left(N_{\text {th }}\right)$ components along the BEC-to-droplet crossover. Since in the droplet regime the core density $n_{\text {core }}(r)$ dramatically increases, 3B losses are expected to play an important role even for moderate and low values of $L_{3}$ [23]. Notwithstanding, 3B losses and QFs exhibit different power dependencies on $n(r)$ [see Eq. (1)] and, thus, the atom-loss dynamics should disclose their competition: while QFs tend to stabilize a high-density state, namely the droplet, 3B losses favor lower densities.

Figures 4(a) and 4(b) show $N_{\text {core }}$ and $N_{\text {th }}$, extracted from the double-Gaussian fit as a function of $a_{s}$ after a nonadiabatic [Fig. 4(a)] and adiabatic [Fig. 4(b)] change of $a_{s}$. Both cases show a similar evolution. When lowering $a_{s}$, $N_{\text {core }}$ is first constant for $a_{s}>a_{\text {dd }}$, then shows a sharp drop starting around $a_{s} \sim a_{\mathrm{dd}}$, and finally curves up for lower $a_{s}$. We note that in the adiabatic case, $N_{\text {core }}$ decreases faster as compared to the nonadiabatic one and finally saturates around $7 \times 10^{3}$ at lower $a_{s}$. We attribute these to the longer timing involved, and we observe a similar trend as well as a similar saturation value for longer $t_{h}$ [see, e.g., Fig. 4(c)].

Remarkably, $N_{\text {th }}$ remains mainly unaltered over the whole range of $a_{s}$ and the whole system does not show any appreciable heating. This suggests that the condensed atoms, which are ejected from in the core, leave the trap instead of being transferred to the thermal component, confirming a picture in which the thermal and the condensed component have uncoupled dynamics.

We now compare the experiment with the theory, which, as previously, precisely accounts for the experimental sequence and its timing by performing RTE along 


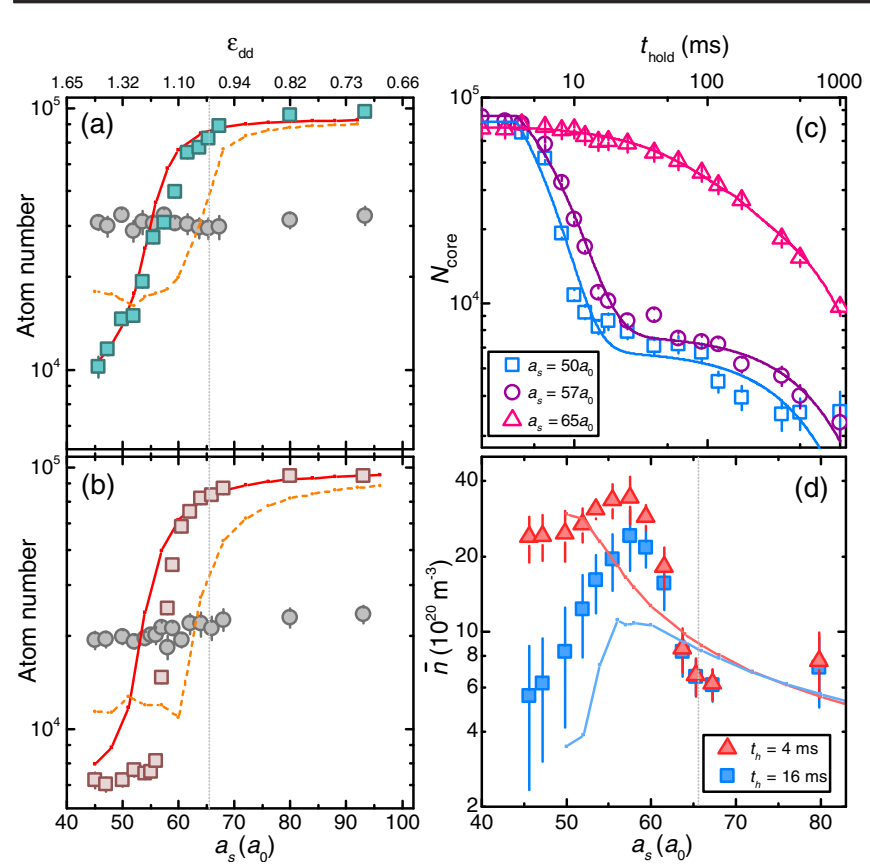

FIG. 4. Lifetime and in situ density of the high-density core. (a), (b) Measured $N_{\text {core }}$ (squares) and $N_{\text {th }}$ (circles) versus $a_{s}$ after (a) a nonadiabatic $\left(t_{r}=10 \mathrm{~ms}, t_{h}=8 \mathrm{~ms}\right)$ and (b) an adiabatic $\left(t_{r}=45 \mathrm{~ms}, t_{h}=0 \mathrm{~ms}\right)$ ramp. The data show a better agreement with the theory with the LHY term (solid line) as compared to the MF theory (dashed line). (c) Time decay of $N_{\text {core }}$ for $a_{s}=65 a_{0}$ (triangles), $57 a_{0}$ (circles), and 50a (squares) after quenching $a_{s}$ $\left(t_{r}=10 \mathrm{~ms}\right)$. We fit a double exponential function to the data (solid lines) [30]. (d) From the fit, we deduct the mean in situ density of the core $\bar{n}$ (see text) for $t_{h}=4 \mathrm{~ms}$ (triangles) and $16 \mathrm{~ms}$ (squares) and as a function of $a_{s}$. The error bars include the statistical errors on the fit and on $L_{3}$. The solid lines show results of the RTE including the LHY correction for $t_{h}=0 \mathrm{~ms}$ (red), $t_{h}=25 \mathrm{~ms}$ (blue).

Eq. (1). Here, we compute the final atom number $N=$ $\int n(\boldsymbol{r}) d^{3} r$ as a function of $a_{s}$ with and without the LHY term. Remarkably, the observed evolution of $N_{\text {core }}$ is very well reproduced by our beyond-MF calculations (solid lines), whereas in the absence of the LHY stabilization, the calculations predict losses in the condensed core to occur at values of $a_{s}$ too large compared to the measured ones; see Figs. 4(a) and 4(b).

The observed evolution of $N_{\text {core }}$ is well reproduced by our beyond-MF calculations (solid lines). The agreement is particularly remarkable for the quench [Fig. 4(a)] while it is slightly degraded in the adiabatic ramp [Fig. 4(b)], with an overestimation of the remaining $N_{\text {core }}$ at small $a_{s}$. This can be explained by noting that, due to the longer time during which the losses set in, a more acute importance is given to $L_{3}$, and by considering the effects of QFs on its value. Indeed, it is of interest to note that many-body effects modify the 3B correlation function $g_{3}$ [44], leading to an enhanced loss rate. This then justifies the larger predicted $N_{\text {core }}$ in our simulation based on the simple noninteracting value $g_{3}=1$ [30] compared to the experiment (we estimate $g_{3} \sim 1.3$ for our typical parameters), and the increased discrepancy with decreasing $a_{s}$, where QFs are doomed to prevail. In contrast, the MF calculations deviate from the experiment with enhanced losses in the $a_{s} \sim a_{\mathrm{dd}}$ region. We note that the abrupt and high saturation of $N_{\text {core }}$ at $a_{s}<a_{\mathrm{dd}}$, distinct from the experimental observations, is a signature of the collapse, reestablishing lower density in the gas via fast "explosive" dynamics.

Finally, we investigate the in-trap time evolution of $N_{\text {core }}$ after quenching $a_{s}$ in the droplet regime; see Fig. 4(c). Our measurements reveal three different time scales for the losses. At very short $t_{h}(\approx 0-3.5 \mathrm{~ms}), N_{\text {core }}$ is roughly constant, which we attribute to the time needed for the high-density state to develop. It follows a fast decay $(\approx 3.5-25 \mathrm{~ms})$, in which the atoms are ejected from the high-density core via 3B losses, and witnesses the formation of a high-density coherent state. The steepness of this fast decay appears to critically depend on $a_{s}$, with a marked acceleration below the MF instability threshold. Then, the loss dynamics substantially slows down $(\approx 25-1000 \mathrm{~ms})$ while a coherent central core is still visible in the density profile (with $N_{\text {core }} \sim 10^{4}$ atoms).

From the loss curves [Fig. 4(c)] and using the general 3B loss relation $\left(1 / N_{\text {core }}\right)\left(\mathrm{d} N_{\text {core }} / d t_{h}\right)=-L_{3} \bar{n}^{2}$, we are able to extract the mean in situ density $\bar{n}=\sqrt{\left\langle n_{\text {core }}(r)^{2}\right\rangle}$ of the high-density component in the BEC-to-droplet crossover. Here, we estimate $N_{\text {core }}\left(t_{h}\right)$ and $\left(\mathrm{d} N_{\text {core }} / d t_{h}\right)$ from an empirical fit to our data and compute $\bar{n}$ using an independent measurement of the 3B loss coefficient $L_{3}$ [30]. Figure 4(d) shows our results for $t_{h}=4,16 \mathrm{~ms}$. We observe a prominent increase of $\bar{n}$ across the threshold $a_{s} \sim a_{\mathrm{dd}}$, and a surviving high-density state deep into the MF instability regime.

The formation of the droplet state is particularly visible for the $t_{h}=4 \mathrm{~ms}$ case. Here, $\bar{n}$ grows from $6.2(9) \times$ $10^{20} \mathrm{~m}^{-3}$ at $a_{s}=67 a_{0}$ to a maximum of $35(7) \times 10^{20} \mathrm{~m}^{-3}$ at $a_{s}=57 a_{0}$, while it is slightly reduced to $\sim 24 \times 10^{20} \mathrm{~m}^{-3}$ at $a_{s} \sim 46 a_{0}$. This direct estimate of $\bar{n}$ advocates the activation of the LHY term when lowering $a_{s}$; additionally, its magnitude as well as its evolution are in good agreement with our simulations including the LHY correction.

Our results together with the good agreement between theory and experiments provide an alternative confirmation of the central role of beyond-mean-field physics. The lifetime of the high-density core reveals, on the one hand, the activation of the LHY term and the crossover toward a dense droplet state, and on the other hand, the counteracting role of 3B losses in regulating the maximum density in the droplet regime.

\section{EXPANSION DYNAMICS}

Besides their dissimilar stability diagram, collective excitations, and density distribution, a dilute BEC in the 
MF regime and a quantum droplet are also expected to exhibit a markedly different expansion dynamics. While the first is confined by an external trapping potential and thus freely expands in its absence, a droplet state is self-bound (SB) by its underlying interaction in analogy with the He-droplet case [20-23]. As in our previous discussions, the evolution from a trap-bound to a self-bound solution is expected to be regulated by the interplay between QFs and 3B loss processes.

We investigate the expansion dynamics of our system for various $a_{s}$. To preserve the high density of the coherent component, our measurements focus on short time scales with $t_{r}=10 \mathrm{~ms}$ and $t_{h}=5 \mathrm{~ms}$. After preparing the system at the desired $a_{s}$, we abruptly switch off the optical dipole trap, let the gas expand for a variable $t_{\mathrm{TOF}}$, and probe the cloud width using the $\|$ imaging. We fit the observed density distribution to a double-Gaussian function, as previously described. To extract the width $\sigma_{\eta}$ of the high-density core $\left(n_{\text {core }}\right)$, we compute the second moments $\sigma_{\eta}^{2}=\int \eta^{2} n_{\text {core }}(\boldsymbol{r}) d \boldsymbol{r}$ along $\eta=x, z$, where $n_{\text {core }}$ is extracted from the double-Gaussian fit. Figure 5(a) exemplifies the TOF evolution of $\sigma_{\eta=x}$ at $a_{s}=93 a_{0}, 64 a_{0}$, and $55.5 a_{0}$. When entering the $\varepsilon_{\mathrm{dd}}>1$ regime, atoms in the highdensity core exhibit a marked slowing-down of the expansion dynamics, which cannot be explained within the MF approach.

To systematically study this effect, we repeat the above measurements for different values of $a_{s}$ (i.e., $\varepsilon_{\mathrm{dd}}$ ). From $\sigma_{\eta}\left(t_{\mathrm{TOF}}\right)$, we extract the value of the expansion velocity $v_{\eta}$ by fitting the data to $\sigma_{\eta}\left(t_{\mathrm{TOF}}\right)=\sqrt{\sigma_{\eta, 0}^{2}+v_{\eta}^{2} t_{\mathrm{TOF}}^{2}}$. Figure 5(b) shows $v_{x}$ in an $\varepsilon_{\mathrm{dd}}$ range from 0.7 to 1.5 . When the system approaches the droplet regime with decreasing scattering length $\left(a_{s}<a_{\mathrm{dd}}\right), v_{x}$ undergoes a strong reduction and drops to a minimum equal to $v_{x}=0.40(2) \mu \mathrm{m} / \mathrm{ms}$ at about $56 a_{0}\left(\varepsilon_{\mathrm{dd}} \sim 1.17\right)$. For further lowering of $a_{s}, v_{x}$ starts to increase again. A similar behavior is observed for $v_{z}$. We note that only the high-density component reveals this intriguing dependency on $a_{s}$, whereas the thermal part shows an almost constant expansion velocity [45].

Considering the fit-free character of our simulations as well as the experimental challenge of accurately estimating the expansion velocities [46], we conclude that our observations agree well with the theory predictions including the LHY term; see solid line in Fig. 5(b). The TOF evolution is calculated using a multigrid numerical scheme [30]. We record the evolution of $\sigma_{\eta}$ with $t_{\mathrm{TOF}}$ and extract the corresponding expansion velocities from the asymptotic behavior of $d \sigma_{\eta} / d t_{\mathrm{TOF}}$. Our simulations show a slowingdown with a minimum of $v_{x}=0.32 \mu \mathrm{m} / \mathrm{ms}$ at $a_{s} \sim 56 a_{0}$ $\left(\varepsilon_{\mathrm{dd}} \sim 1.17\right)$, followed by an increase at lower $a_{s}$. In contrast, calculations in the absence of beyond-MF corrections fail to reproduce the experimental data. Here, the velocity is first slightly more reduced above the MF instability threshold $\varepsilon_{\mathrm{dd}} \sim 1$ than is expected with LHY
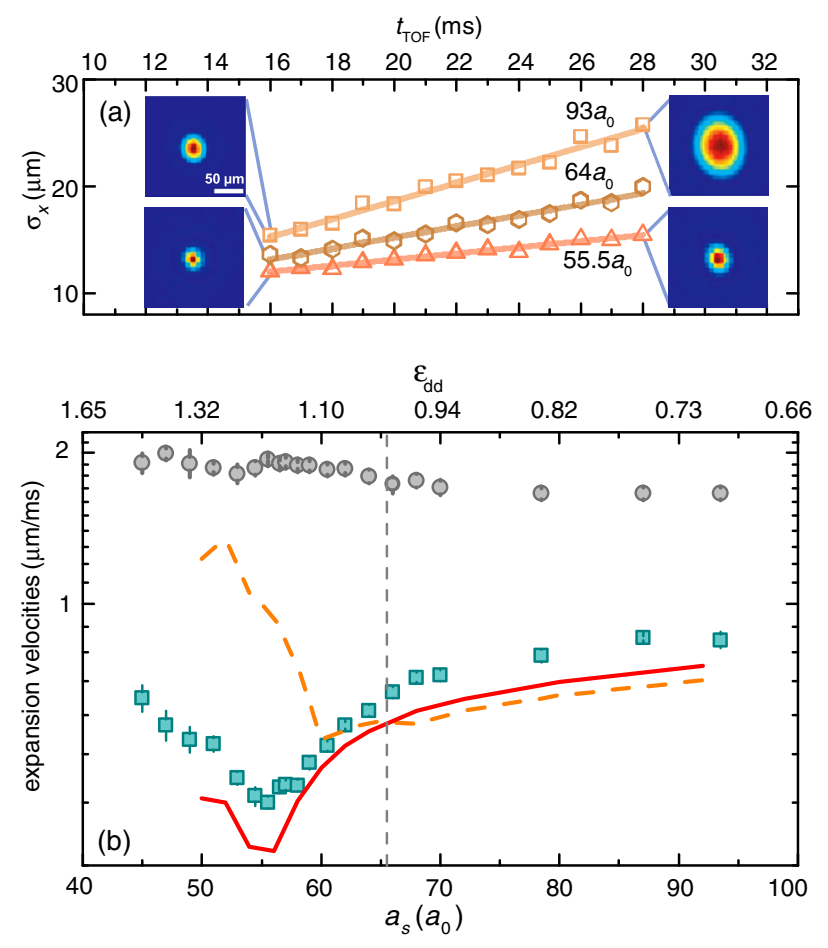

FIG. 5. Expansion dynamics across the BEC-to-droplet crossover. (a) TOF evolution of the width $\sigma_{x}$ of the high-density component for $a_{s}=93 a_{0}$ (squares), $64 a_{0}$ (circles), $55.5 a_{0}$ (triangles). The lines are fit to the data using $\sigma_{x}\left(t_{\mathrm{TOF}}\right)=\sqrt{\sigma_{x, 0}^{2}+v_{x}^{2} t_{\mathrm{TOF}}^{2}}$, from which we extract $v_{x}$. (b) $v_{x}$ as a function of $a_{s}$ (squares). For comparison, the $a_{s}$-independent expansion velocities of the thermal component are also shown (circles). The experimental data are in very good agreement with our parameter-free theory from RTE simulations including the LHY term (solid line) and rule out the MF scenario (dotted line). For clarity, we show only $v_{x}$; similar results are found for $v_{z}$.

corrections, it then already increases at this threshold. The first point relies on the trivial slowing-down of a BEC whose mean repulsion energy is weakened (by reducing $a_{s}$ or decreasing its population $\left.N_{\text {core }}\right)$. The second point reveals a collapsing behavior that gives rise to an explosive evolution of the density profile. The minimal velocity is found here to be $v_{x}=0.56 \mu \mathrm{m} / \mathrm{ms}$ at $a_{s}=68 a_{0}$, which is a much higher value than both our experimental results and our theory predictions including the LHY correction.

The expansion behavior can be qualitatively well understood considering the so-called released, or internal, energy $E_{R}$. This is the energy of the system when subtracting the energy related to the confinement [1]. In the MF scenario, $E_{R}>0$, as long as the ground state is stable. The BEC expands ballistically and $v_{\eta}$ decreases for decreasing $a_{s}$ and $N$. In the unstable regime, the expansion velocity depends crucially on the value of $t_{h}$ at which the trap is switched off due to the occurrence of an in situ collapse dynamic. On the contrary, in the presence of $\mathrm{QF}$, a stable ground state always exists. The sharp variability in $t_{h}$ is expected to be 
suppressed. Assuming a fixed $N_{\text {core }}$ (i.e., no 3B losses), one can show that $E_{R}$ decreases with decreasing $a_{s}$ and eventually reaches $E_{R}<0$ for $a_{s}<a_{\mathrm{SB}}$, marking the onset of the SB solution (e.g., $a_{\mathrm{SB}}=56 a_{0}$ for $N=1.2 \times 10^{5}$ ) [30]. However, in stark contrast to the MF case, $E_{R}$ increases with decreasing $N_{\text {core }}$ in the droplet regime. We note that $a_{\mathrm{SB}}$ is then shifted to lower values when $N_{\text {core }}$ gets reduced by $3 \mathrm{~B}$ losses, thus affecting the lifetime of the self-bound solution.

The existence of a minimal expansion velocity is thus a direct consequence of the competition between the decrease of $E_{R}$ for decreasing $a_{s}$ at a fixed $N_{\text {core }}$ and the increase of $E_{R}$ for decreasing $N_{\text {core }}$ in the droplet regime. In the crossover regime, the system smoothly evolves towards a fully selfbound state $\left(v_{\eta}=0\right)$ until 3B losses, occurring in the trap or in the initial phase of the expansion, set in to unbind the system and to reduce the lifetime of the droplet state.

\section{CONCLUSION}

In summary, we demonstrate the existence of the crossover from a dilute BEC to a quantum droplet state driven by QFs. Our experiments not only demonstrate that LHY stabilization is a general feature of strongly dipolar gases, but also thoroughly investigate the driving role of QFs in dictating the system properties, in particular, its collective mode, its atom losses, and expansion dynamics. This clear and quantitative demonstration of the impact of QFs in dipolar gases intrinsically relies on our unique and precise knowledge of $a_{s}$ that alone enables a direct comparison to a parameter-free theory, which is based on a generalized GPE with LHY correction. Our combined experimental and theoretical results ultimately offer an experimental validation of the modeling proposed in Ref. [20] and thus of the latter results of Refs. [21-23].

\section{ACKNOWLEDGEMENT}

We thank M. Baranov, R. van Bijnen, R. N. Bisset, B. Blakie, E. Demler, R. Grimm, T. Pfau, and the Stuttgart Dy team for multiple useful discussions. We thank G. Faraoni for her support in the final stage of the experiment. The Innsbruck group thanks the European Research Council within the ERC Consolidator Grant RARE No. 681432. L.C. is supported within the Marie Curie Project DIPPHASE No. 706809 of the European Commission. The Hannover group acknowledges the support of the DFG (RTG 1729). Both groups acknowledge the support of the DFG/FWF (FOR 2247).

Note added in the proof.-A related work by M. Schmitt et al. recently appeared [47].

[1] L. Pitaevskii and S. Stringari, Bose-Einstein Condensation (Oxford University Press, Oxford, England, 2003).
[2] C. Chin, R. Grimm, P.S. Julienne, and E. Tiesinga, Feshbach Resonances in Ultracold Gases, Rev. Mod. Phys. 82, 1225 (2010).

[3] T. D. Lee, K. Huang, and C. N. Yang, Eigenvalues and Eigenfunctions of a Bose System of Hard Spheres and Its Low-Temperature Properties, Phys. Rev. 106, 1135 (1957).

[4] A. Altmeyer, S. Riedl, C. Kohstall, M. J. Wright, R. Geursen, M. Bartenstein, C. Chin, J. H. Denschlag, and R. Grimm, Precision Measurements of Collective Oscillations in the BEC-BCS Crossover, Phys. Rev. Lett. 98, 040401 (2007).

[5] Y.-i. Shin, A. Schirotzek, C. H. Schunck, and W. Ketterle, Realization of a Strongly Interacting Bose-Fermi Mixture from a Two-Component Fermi Gas, Phys. Rev. Lett. 101, 070404 (2008).

[6] N. Navon, S. Nascimbène, F. Chevy, and C. Salomon, The Equation of State of a Low-Temperature Fermi Gas with Tunable Interactions, Science 328, 729 (2010).

[7] S. B. Papp, J. M. Pino, R. J. Wild, S. Ronen, C. E. Wieman, D. S. Jin, and E. A. Cornell, Bragg Spectroscopy of a Strongly Interacting ${ }^{85} \mathrm{Rb}$ Bose-Einstein Condensate, Phys. Rev. Lett. 101, 135301 (2008).

[8] N. Navon, S. Piatecki, K. Günter, B. Rem, T. C. Nguyen, F. Chevy, W. Krauth, and C. Salomon, Dynamics and Thermodynamics of the Low-Temperature Strongly Interacting Bose Gas, Phys. Rev. Lett. 107, 135301 (2011).

[9] D. S. Petrov, Quantum Mechanical Stabilization of a Collapsing Bose-Bose Mixture, Phys. Rev. Lett. 115, 155302 (2015).

[10] T. Lahaye, C. Menotti, L. Santos, M. Lewenstein, and T. Pfau, The Physics of Dipolar Bosonic Quantum Gases, Rep. Prog. Phys. 72, 126401 (2009).

[11] M. A. Baranov, M. Dalmonte, G. Pupillo, and P. Zoller, Condensed Matter Theory of Dipolar Quantum Gases, Chem. Rev. 112, 5012 (2012).

[12] T. Koch, T. Lahaye, J. Metz, B. Fröhlich, A. Griesmaier, and T. Pfau, Stabilization of a Purely Dipolar Quantum Gas against Collapse, Nat. Phys. 4, 218 (2008).

[13] T. Lahaye, J. Metz, B. Fröhlich, T. Koch, M. Meister, A. Griesmaier, T. Pfau, H. Saito, Y. Kawaguchi, and M. Ueda, $d$-Wave Collapse and Explosion of a Dipolar Bose-Einstein Condensate, Phys. Rev. Lett. 101, 080401 (2008).

[14] J. Metz, T. Lahaye, B. Fröhlich, A. Griesmaier, T. Pfau, H. Saito, Y. Kawaguchi, and M. Ueda, Coherent Collapses of Dipolar Bose-Einstein Condensates for Different Trap Geometries, New J. Phys. 11, 055032 (2009).

[15] H. Kadau, M. Schmitt, M. Wenzel, C. Wink, T. Maier, I. Ferrier-Barbut, and T. Pfau, Observing the Rosensweig Instability of a Quantum Ferrofluid, Nature (London) 530, 194 (2016).

[16] I. Ferrier-Barbut, H. Kadau, M. Schmitt, M. Wenzel, and T. Pfau, Observation of Quantum Droplets in a Strongly Dipolar Bose Gas, Phys. Rev. Lett. 116, 215301 (2016).

[17] K.-T. Xi and H. Saito, Droplet Formation in a Bose-Einstein Condensate with Strong Dipole-Dipole Interaction, Phys. Rev. A 93, 011604 (2016).

[18] R. N. Bisset and P.B. Blakie, Crystallization of a Dilute Atomic Dipolar Condensate, Phys. Rev. A 92, 061603 (2015). 
[19] P. B. Blakie, Properties of a Dipolar Condensate with Three-Body Interactions, Phys. Rev. A 93, 033644 (2016).

[20] F. Wächtler and L. Santos, Quantum Filaments in Dipolar Bose-Einstein Condensates, Phys. Rev. A 93, 061603 (2016).

[21] R. N. Bisset, R. M. Wilson, D. Baillie, and P. B. Blakie, Ground State Phase Diagram of a Dipolar Condensate with Quantum Fluctuations, Phys. Rev. A 94, 033619 (2016).

[22] F. Wächtler and L. Santos, Ground-State Properties and Elementary Excitations of Quantum Droplets in Dipolar Bose-Einstein Condensates, Phys. Rev. A 94, 043618 (2016).

[23] D. Baillie, R.M. Wilson, R.N. Bisset, and P.B. Blakie, Self-Bound Dipolar Droplet: A Localized Matter Wave in Free Space, Phys. Rev. A 94, 021602(R) (2016).

[24] Note that Er offers a system of intermediate dipolar strength $\left(a_{\mathrm{dd}}=65.5 a_{0}\right)$ between $\mathrm{Cr}\left(a_{\mathrm{dd}}=15 a_{0}\right)$ and Dy $\left(a_{\mathrm{dd}}=132 a_{0}\right)$.

[25] A. Frisch, M. Mark, K. Aikawa, F. Ferlaino, J. L. Bohn, C. Makrides, A. Petrov, and S. Kotochigova, Quantum Chaos in Ultracold Collisions of Gas-Phase Erbium Atoms, Nature (London) 507, 475 (2014).

[26] S. Baier et al. (to be published).

[27] K. Aikawa, A. Frisch, M. Mark, S. Baier, A. Rietzler, R. Grimm, and F. Ferlaino, Bose-Einstein Condensation of Erbium, Phys. Rev. Lett. 108, 210401 (2012).

[28] S. Baier, M. J. Mark, D. Petter, K. Aikawa, L. Chomaz, Z. Cai, M. Baranov, P. Zoller, and F. Ferlaino, Extended BoseHubbard Models with Ultracold Magnetic Atoms, Science 352, 201 (2016).

[29] M. J. Mark, E. Haller, K. Lauber, J. G. Danzl, A. J. Daley, and H.-C. Nägerl, Precision Measurements on a Tunable Mott Insulator of Ultracold Atoms, Phys. Rev. Lett. 107, 175301 (2011).

[30] See Supplemental Material at http://link.aps.org/ supplemental/10.1103/PhysRevX.6.041039 for further details on our experimental setup and procedure, on the data analysis performed, on our numerical simulations and on additional calculations using the Gaussian ansatz.

[31] J. L. Roberts, N. R. Claussen, S. L. Cornish, E. A. Donley, E. A. Cornell, and C. E. Wieman, Controlled Collapse of a Bose-Einstein Condensate, Phys. Rev. Lett. 86, 4211 (2001).

[32] E. A. Donley, N. R. Claussen, S. L. Cornish, J. L. Roberts, E. A. Cornell, and C. E. Wieman, Dynamics of Collapsing and Exploding Bose-Einstein Condensates, Nature (London) 412, 295 (2001).

[33] M. Ueda and H. Saito, A Consistent Picture of a Collapsing Bose-Einstein Condensate, J. Phys. Soc. Jpn. 72, 127 (2003).

[34] R. Grimm, in Ultra-Cold Fermi Gases, Proceedings of the International School of Physics "Enrico Fermi", Course CLXIV, edited by M. Inguscio, W. Ketterle, and C. Salomon (2007) [arXiv:cond-mat/0703091].

[35] L. Pitaevskii and S. Stringari, Elementary Excitations in Trapped Bose-Einstein Condensed Gases beyond the
Mean-Field Approximation, Phys. Rev. Lett. 81, 4541 (1998).

[36] $\nu_{\|}$is determined from the center-of-mass oscillations along $y$, which are simultaneously excited with the axial breathing mode.

[37] G. Bismut, B. Pasquiou, E. Maréchal, P. Pedri, L. Vernac, O. Gorceix, and B. Laburthe-Tolra, Collective Excitations of a Dipolar Bose-Einstein Condensate, Phys. Rev. Lett. 105, 040404 (2010).

[38] We indeed observe a high-energy mode in which the highdensity core splits and recombines in a damped oscillating manner.

[39] A. R.P. Lima and A. Pelster, Quantum Fluctuations in Dipolar Bose Gases, Phys. Rev. A 84, 041604 (2011).

[40] A. R. P. Lima and A. Pelster, Beyond Mean-Field LowLying Excitations of Dipolar Bose Gases, Phys. Rev. A 86, 063609 (2012).

[41] A. Boudjemâa, Theory of Excitations of Dipolar BoseEinstein Condensate at Finite Temperature, J. Phys. B 48, 035302 (2015).

[42] We note that the use of a generalized GPE, valid in the TF regime, can be extended beyond this regime as long as the LHY correction is dominated by short-wavelength excitations, whose momentum $\boldsymbol{q}$ fulfills $q R \gg 1$, where $R$ is the size of the condensate along the direction of $q$. As discussed in detail in Refs. [20,22], this is the case for large droplet and $\varepsilon_{\mathrm{dd}}$ close to 1 . Our experiments, performed in cigarshaped traps with relatively large BECs, offer privileged conditions to be compared to generalized GPE predictions (in particular, enhanced compared to Refs. [15,16]). Indeed, we quantitatively estimate the validity of this approximation in our study by introducing an anisotropic cutoff, $q_{c}(\theta)=q_{y} \sqrt{\cos ^{2}(\theta)+K^{2} \sin ^{2}(\theta)}$, with $K$ the aspect ratio of the BEC, assuming here an isotropic geometry around the dipole axis $y$, and $\theta$ denoting the angle $(y, \boldsymbol{q})$ [22]. For typical experimental parameters, e.g., $a_{s}=58 a_{0}, N=$ $5 \times 10^{4}$, we find an axial radius $R_{y}=13 \mu \mathrm{m}$ and $K=7.5$. Introducing a typical cutoff value $q_{y} R_{y}=25$, the cutoff LHY correction [i.e., taking momenta $q>q_{c}(\theta)$ ] equals $\sim 0.8$ of the value calculated in homogeneous space, demonstrating that short-wavelength excitations indeed have a dominant contribution and validating our approach. We note that, when decreasing $a_{s}$, longwavelength excitations and unstable modes come to play a more significant role, and for this reason we consider our simulation reliable down to $a_{s} \geq 50 a_{0}$.

[43] Yu. Kagan, A. E. Muryshev, and G. V. Shlyapnikov, Collapse and Bose-Einstein Condensation in a Trapped Bose Gas with Negative Scattering Length, Phys. Rev. Lett. 81, 933 (1998).

[44] Yu Kagan, B. V. Svistunov, and G. V. Shlyapnikov, Effect of Bose Condensation on Inelastic Processes in Gases, Pis'ma Zh. Eksp. Teor. Fiz. 42, 169 (1985).

[45] We observe a $14(9) \%$ increase of $v_{\text {th }}$ over the $a_{s}$ range when decreasing $a_{s}$ that we attribute to fit artifacts. First, the double Gaussian fit does not properly account either for the coherent or for the thermal parts of the gas which may result in cross talk in the fit of these components. Second, flaws in the partition in two components may occur, especially for 
the configuration where both components have similar sizes (short TOF). We note that at long $t_{\mathrm{TOF}}$ the thermal sizes are nearly constant, while a trend in their evolution with $a_{s}$ appears at short $t_{\mathrm{TOF}}$. Because they are less subject to artifacts, the long $t_{\mathrm{TOF}}$ are expected to more reliably estimate $v$ and we hence conclude that the observed slight heating is, at least mainly, an artifact.

[46] The observed slight overall shift of the experimental data compared to the theoretical predictions may be attributed to experimental artifacts coming from (i) the restricted range of
$t_{\mathrm{TOF}}$ experimentally accessible, (ii) artifacts of the expansion fit arising, in particular, from the interplay of the two fit parameters, the empirical expression of the fit, or the finite range of expansion time [cf. (i)], or (iii) artifacts coming from the empirical double Gaussian fit used here to extract $\sigma$. This may lead to misassessment of the size of the coherent part and of its evolution with $t_{\mathrm{TOF}}$.

[47] M. Schmitt, M. Wenzel, F. Böttcher, I. Ferrier-Barbut, T. Pfau, Self-Bound Droplets of a Dilute Magnetic Quantum Liquid, Nature (London) 539, 259 (2016). 